Proceedings of the International School and Conference on Optics and Optical Materials, ISCOM07, Belgrade, Serbia, September 3-7, 2007

\title{
High Resolution Spectroscopy of Cold, Trapped Atoms
}

\author{
T.M. Brzozowski, M. Brzozowska, J. Zachorowski \\ AND W. GAWLIK \\ Marian Smoluchowski Institute of Physics, Jagiellonian University \\ Reymonta 4, 30-059 Cracow, Poland

\begin{abstract}
Pump-probe spectroscopy of cold, trapped atoms is discussed with particular attention to mechanisms specific for cold atoms and potential diagnostics applications. The discussion is illustrated with experimental results obtained with ${ }^{85} \mathrm{Rb}$ atoms trapped in a magneto-optical trap. Most important applications are non-destructive, real-time velocimetry (thermometry) and analysis of optical lattice dynamics.
\end{abstract}

PACS numbers: $32.80 . \mathrm{Pj}, 42.50 . \mathrm{Vk}, 42.65 .-\mathrm{k}$

\section{Introduction}

Cold atoms in atomic traps are attractive samples for many important experiments. One of few available methods for their diagnostics is laser spectroscopy. In this paper we present typical spectra obtained by nonlinear Raman pump-probe spectroscopy and discuss their main features and applications.

In our experiment we use a standard vapor-loaded magneto-optical trap (MOT). In order to perform high resolution Raman spectroscopy one of the lasers serves as the master for injection seeding into the trapping, pump and probe lasers. Their frequency shifts are controlled by acousto-optic modulators (AOMs). The probe beam enters the cloud of cold atoms making a small angle $\theta$ with one of the trapping beams. After traversing the cloud, the probe beam is directed onto a photodiode which records the absorption spectrum as a function of the pump-probe detuning, $\delta=\omega_{\mathrm{pr}}-\omega$.

The example of the probe absorption is presented in Fig. 1a. The spectrum consists of a narrow, central resonance for pump-probe detuning $\delta \approx 0$ and two side-band resonances. Such a shape can be qualitatively understood in terms of a two-level dressed atom model [1] extended to include multilevel structure of rubidium states and inhomogeneities of the net optical field in the trap. The results of such modeling [2] are in a good agreement with the experimental signals. 


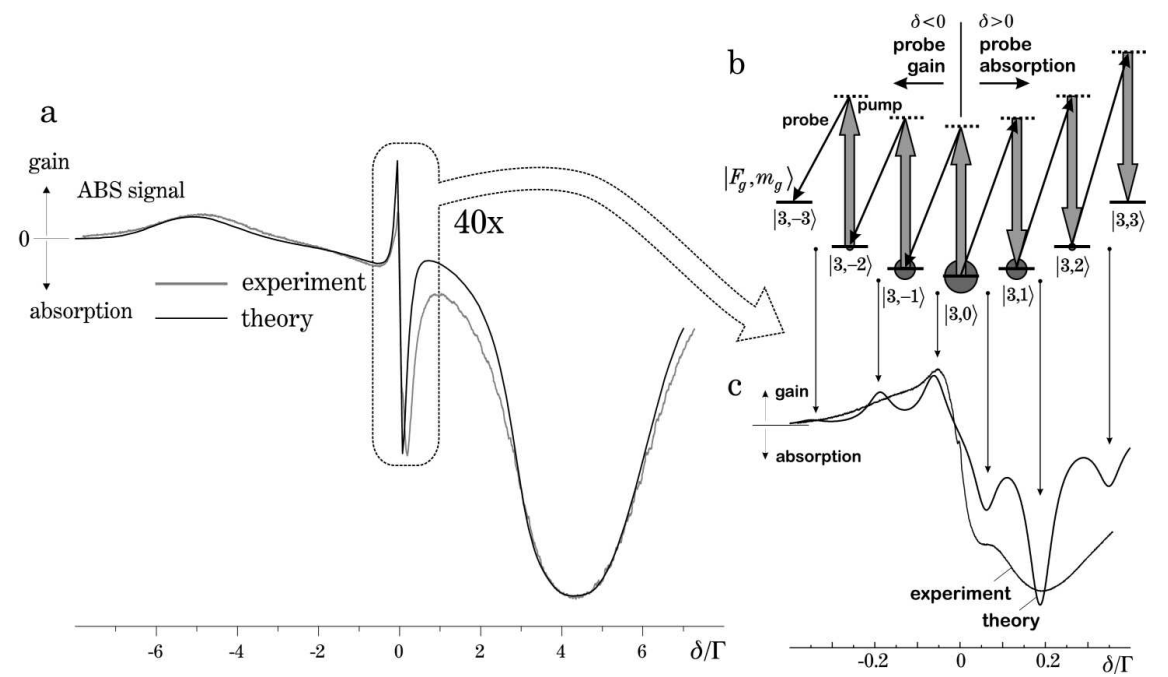

Fig. 1. (a) Experimental absorption spectra (gray lines) and their modeling (black lines) [2]. By narrowing the probe scan we can record the central feature with higher resolution (c). The shape of this feature is explained in parts (b) and (c). (b) Multilevel structure of the $5{ }^{2} S_{1 / 2}(F=3){ }^{85}$ Rb ground state. Zeeman sublevels are perturbed with a $\pi$-polarized pump (thick grey arrows), which results in their energy shift and symmetric population distribution (alignment), marked by grey circles. The structure is then probed by a $\sigma^{+}$-polarized beam. (c) Theoretical absorption signal (black) compared with the experimental spectrum (gray). The theoretical curve was generated for the Rabi frequency $\Omega=6 \Gamma$, detuning $\Delta=-3 \Gamma$. Experimental conditions: $I_{\max }=10.2 \mathrm{~mW} / \mathrm{cm}^{2}$, $\Delta=-3 \Gamma, \partial B / \partial z=13 \mathrm{Gs} / \mathrm{cm}$. The discrepancy between the simple model and experiment is primarily due to the lack of inclusion of $\boldsymbol{E}$ and $\boldsymbol{B}$-field inhomogeneities in the trap [2].

By narrowing the probe frequency range one sees that the resonance at $\omega_{\mathrm{pr}} \simeq \omega$ (for $\delta \simeq 0$ ) comprises many different contributions (Fig. 1, inset b), not explained by the dressed atom model. They originate from the Raman transitions between light-shifted sublevels of the ${ }^{2} S_{1 / 2}\left(F_{\mathrm{g}}=3\right)$ level of ${ }^{85} \mathrm{Rb}$, involving one pump photon from the trapping beams and one photon from the probe beam. These transitions are resonant whenever $|\delta|$ coincides with the energy separation of the adjacent sublevels. In order to model the spectra one also has to include $\boldsymbol{E}$ and $\boldsymbol{B}$-field inhomogeneities in the trap. However, such a modeling [2], despite its better agreement with experimental signal for $\delta \approx 0$ still does not provide the exact shape of the absorption curve in its very central part, namely for $|\delta|<0.05 \Gamma$. This can only be achieved by including the processes of the momentum exchange between atoms and the laser field, leading to so-called recoil-induced resonances (RIR) [3-7]. 


\section{Theory}

An atom of mass $m$ interacting with a weak probe beam (wave vector $\boldsymbol{k}_{\mathrm{pr}}$, frequency $\omega+\delta$ ), which makes an angle $\theta$ with a strong pump beam (wave vector $\boldsymbol{k}$, frequency $\omega$ ) (Fig. 2a) undergoes Raman transition between the atomic momentum states. The resonance occurs whenever the probe-pump detuning coincides with the kinetic energy difference and results in atomic momentum change $\Delta p$. Integration over all possible momentum values (Maxwellian distribution for the gas temperature $T$ ) leads to the formula for the $\operatorname{RIR}$ signal $[8,5]$ :

$$
s_{\mathrm{RIR}}(\delta, \theta)=-\sqrt{\frac{m}{2 \pi}} \frac{\hbar \delta}{2 u_{T}^{3 / 2} k \sin (\theta / 2)} \exp \left(-\frac{\delta^{2}}{2 u_{T}^{2}(2 k \sin (\theta / 2))^{2}}\right),
$$

where $u_{T}=\sqrt{k_{\mathrm{B}} T / m}$ is the most probable atomic speed. Signal (1) is the derivative of a Gaussian function. Since the width $\Delta_{\mathrm{RIR}}$ of the signal is proportional to $\sqrt{T}$, the RIR spectrum can serve as a spectroscopic tool for the temperature measurement of a cold atomic sample [4, 9, 10], according to the formula

$$
T=\frac{m}{16 k_{\mathrm{B}} k^{2} \sin (\theta / 2)} \Delta_{\mathrm{RIR}}^{2}
$$

a)

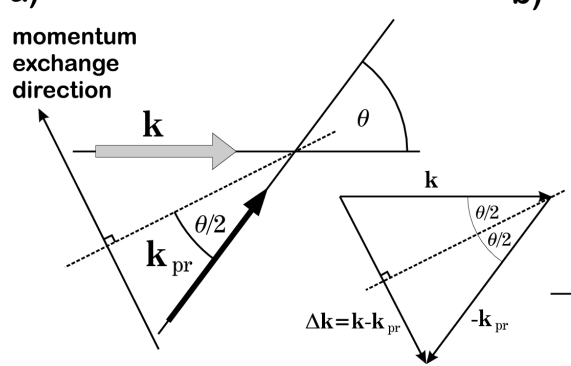

b)

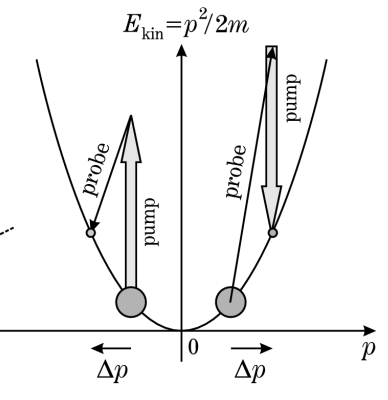

Fig. 2. (a) Basic geometry for the recoil induced resonance, associated with atomic interaction with two beams: the pump (thick gray arrow) and probe (thin arrow) intersecting in an atomic sample at a small angle $\theta$. (b) Raman transitions between the kinetic momentum states for an atom in a state with specific magnetic quantum number.

For systems in a thermodynamic equilibrium, $T$ is a well defined kinetic temperature. If, however, the cold-gas sample is not in thermal equilibrium state, the width of the RIR associated with the momentum transfer in a given direction reflects just the width $\tau$ of a given component of the momentum distribution. To successfully implement the RIRs as a tool for studying the kinetics of the cold atoms, one has to eliminate the Raman-Zeeman resonances (RZRs) contribution from the absorption spectra. 


\section{Experimental}

We have developed a method for observation of a solitary RIR nonoverlapping with RZRs. The method employs an extra pump beam, detuned from the atomic transition by $\delta^{\prime}=+23 \Gamma$ in the described experiments, and scanning the probe beam frequency around the frequency of the new pump (Fig. 3a). Such a solution decouples the Raman spectroscopy from the not well-controlled trapping field and enables non-destructive spectroscopic measurement that can be carried out independently of the MOT action. Moreover, the control over polarization of the extra pump simplifies theoretical description of the observed spectra and allows one to use the theory outlined above.
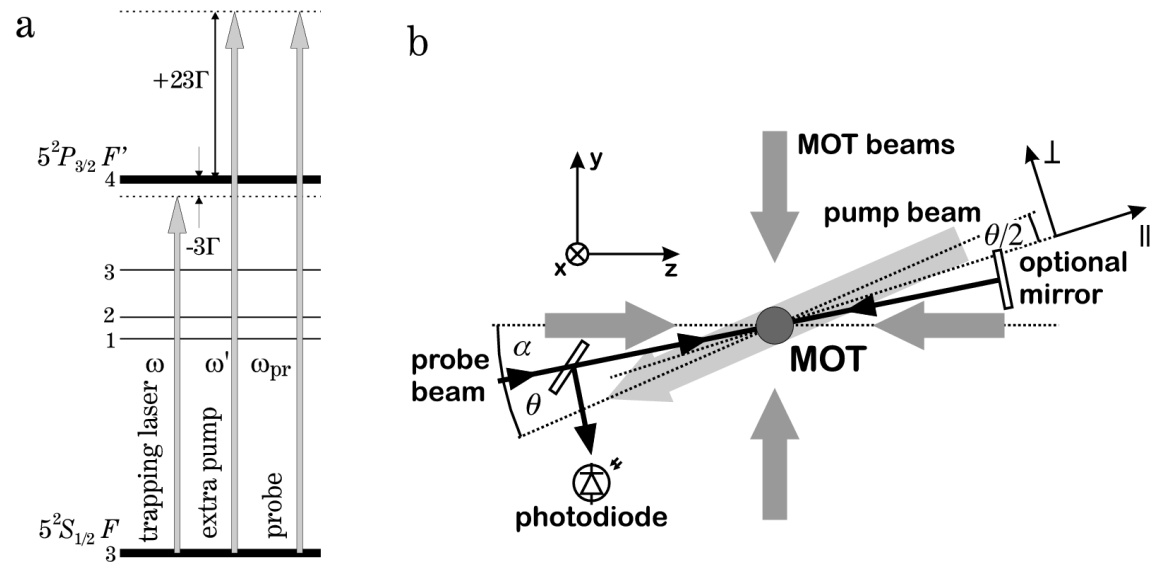

Fig. 3. (a) The laser frequency scheme with an extra pump for RIR Raman spectroscopy. (b) Experimental layout: the pump and probe beams intersect in a cloud of cold atoms. With the optional mirror we realize a three-beam configuration in which the momentum exchange is allowed in two directions: $\perp$ and $\|$ (Third pair of the MOT beams along $x$ and the MOT coils are not shown.).

The new approach to RIR measurements allowed us to measure temperature of MOT as a function of various trapping parameters, to detect and measure the anisotropy of momentum distribution in the unbalanced trap [8] and to create and diagnose 1D optical lattices superimposed on the cloud of cold atoms in a working MOT [11].

For the non-destructive study of nonequilibrium states of the trap, the probe beam is directed at a small angle $\alpha=3^{\circ}$ to one MOT beam (propagating along $z$ ), and the extra pump is at $\theta=5^{\circ}$ to the probe (Fig. 3b). The probe beam can be detected either directly or after retroreflection from the optional mirror. The setup with the retroreflected probe enables the measurement of the momentum distribution $\Pi(\boldsymbol{p})$ simultaneously along two perpendicular directions: $\perp$, for angle $\theta$ between the pump and the nearly co-propagating probe, and $\|$, for angle $180^{\circ}-\theta$ 
between the pump and the nearly counter-propagating probe. When $\alpha$ and $\theta$ are sufficiently small, $\perp$ and $\|$ almost coincide with the $y$ and $z$ directions, respectively.

\section{Discussion}

Typical example of the retroreflected-probe transmission spectrum is shown in Fig. 4a and comprises two contributions. The narrow one results from the Raman process involving the pump and the probe beams making small angle $\theta$ and the wide one is for angle $180^{\circ}-\theta$.
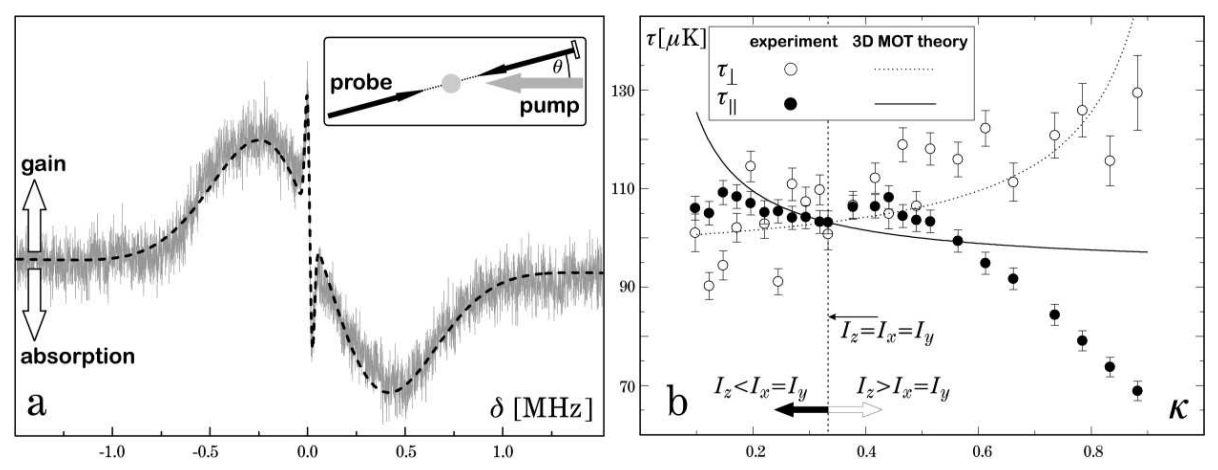

Fig. 4. (a) Transmission spectrum of a retroreflected probe (gray) and the theoretical prediction (dashed). Inset: the beam setup. Experimental conditions: $I_{\mathrm{MOT}}=$ $13.8 \mathrm{~mW} / \mathrm{cm}^{2}$ per beam, $\Delta_{\mathrm{MOT}}=-3 \Gamma, I_{\mathrm{REP}}=15 \mathrm{~mW} / \mathrm{cm}^{2}, \partial_{x} B=12 \mathrm{Gs} / \mathrm{cm}$, the pump beam intensity $I=33 \mathrm{~mW} / \mathrm{cm}^{2}$, and the probe beam intensity $0.3 \mathrm{~mW} / \mathrm{cm}^{2}$. A weak imbalance of the retroreflected trapping beam intensities is responsible for the shift of the resonance centers [8]. (b) Widths of the kinetic momentum distributions (in $\mu \mathrm{K}$ ) measured as a function of relative intensity $\kappa$ (defined in the text) in the two perpendicular directions, $\tau_{\perp}$ (hollow circles) and $\tau_{\|}$(filled circles). For the equilibrium $(\kappa=1 / 3), I_{\mathrm{MOT}}=6.8 \mathrm{~mW} / \mathrm{cm}^{2}, \tau_{\perp}=100 \pm 3 \mu \mathrm{K}$, and $\tau_{\|}=103 \pm 2 \mu \mathrm{K}$. Other parameters as in part (a). Theoretical curves are plotted according to Refs. [12, 13].

As the velocity distributions derived from the signal are Maxwellian, one can determine the values $\tau_{\perp}=172 \pm 6 \mu \mathrm{K}$ and $\tau_{\|}=170 \pm 3 \mu \mathrm{K}$. The equality of these $\tau \mathrm{s}$ implies thermodynamical equilibrium and allows their interpretation as temperature $T$.

When the trapping light is unevenly distributed between the MOT beam pairs the thermodynamics of the system becomes non-trivial. For example, under such conditions, the width of kinetic momentum distribution may show directional dependence [12]. To observe such non-equilibrium state of the cold-atom cloud we changed intensity balance between the longitudinal $\left(I_{z}\right)$ and transverse $\left(I_{x}, I_{y}\right)$ MOT beam pairs, while keeping the total intensity $I_{0}=I_{x}+I_{y}+I_{z}$ constant. We define parameter $\kappa$ as the relative intensity of $I_{z}, I_{z}=\kappa I_{0}, I_{x}=I_{y}=(1-$ $\kappa) I_{0} / 2$. The results of the measurement of $\tau_{\|}$and $\tau_{\perp}$ for different values of $\kappa$ are 
depicted in Fig. 4b. For equal partition of the trapping intensity $(\kappa=1 / 3)$, the widths of kinetic momentum distributions are the same, as expected. However, when $\kappa$ increases, $\tau_{\|}$and $\tau_{\perp}$ follow opposite trends, which is evidence of a kinetic momentum anisotropy in a working MOT and thereby its non-equilibrium state.

The theoretical behavior of $\tau_{\|}$and $\tau_{\perp}$ according to Refs. $[12,13]$ is plotted in Fig. 4b along with the experimental data. They exhibit similar qualitative dependence (the decrease in $\tau_{\|}$and the increase in $\tau_{\perp}$ with growing $\kappa$ ). Remaining discrepancy is due to additional mechanism of sub-Doppler cooling, not included in the model of Ref. [12].

Our experimental setup allows feasible creation of the robust 1D optical lattice in a MOT and its non-destructive diagnostics by RIRs. For this sake, instead of retroreflecting the probe beam, one has to reflect the extra pump beam. In such a configuration, there are also two different angles between probe and one of the pump beams, $\theta$ and $180^{\circ}-\theta$ and, consequently, two directions of the possible momentum transfer. However, in contrast to the case in which we measure the momentum anisotropy, the intense retroreflected pump beam constitutes a standing wave with a modulated-intensity pattern which serves as an 1D optical lattice for the atoms. As a result, the atomic motion in one direction becomes thus restricted to the potential wells of the size of $\lambda / 2$ and hence quantized. This results in vibrational structure of energy levels and leads to the Raman vibrational resonances in the absorption spectrum [5, 14]. Figure 5 presents a series of measurements taken for different temperatures of the atomic cloud. At temperatures below the Doppler limit, $T_{\mathrm{D}}=140 \mu \mathrm{K}$, atoms localized in lattice wells become a significant fraction of the whole sample and give rise to a pronounced vibrational structure. At the same time, they do not generate to the wide RIR signal associated with movement in the $\hat{\boldsymbol{e}}_{\|}$direction but they participate only in the generation of narrow RIR and Raman vibrational spectrum. Such a suppression of recoil in an optical lattice, consistent with the previous predictions [3, 15-17] and experiments $[5,18]$ leads to a novel method for diagnostics of lattice loading by measuring the magnitude of the appropriate RIR signal, as presented in detail in [11].

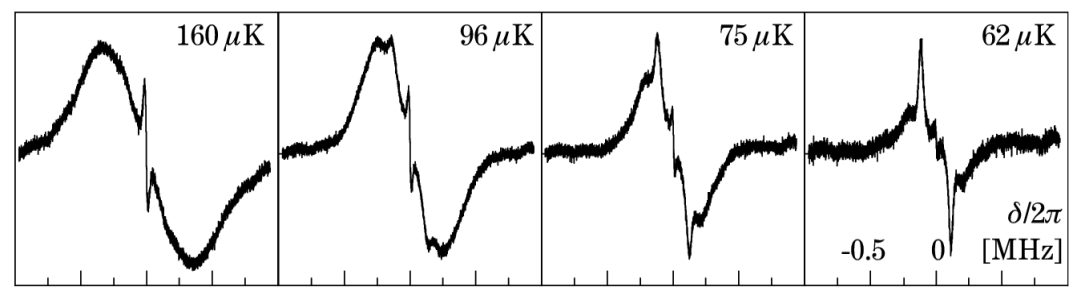

Fig. 5. Comparison of the probe transmission spectra for various MOT-beam temperatures. For sufficiently low temperatures the wide RIR contribution disappears while the lattice contribution becomes distinct [11]. 
Similar spectroscopy methods can be applied to quantum degenerate gas samples, e.g. the Bose-Einstein condensates (BEC). Because of high sensitivity of BEC gases to external perturbations, the pump and probe beams need to be strongly non-resonant. The technique is known as the Bragg spectroscopy and, in fact, is nothing but the Raman (RIR) spectroscopy discussed above. Two counterpropagating beams of slightly different frequencies drive two-photon Raman transitions between atomic kinetic states in the BEC and the resulting momentum kick $2 \hbar k$ splits the condensate into two fractions. Because of the small number of atoms in a condensate, the probe optical signals (absorption and/or dispersion) are usually too weak to be detected directly. More practical detection method is optical imaging, which is most often performed with resonant light beams, so it is destructive, but the non-destructive imaging techniques are also used [19]. Imaging the split atomic cloud of a BEC expanding in gravity allows to determine the number of atoms which underwent Raman transition between two momentum states. This kind of the Bragg spectroscopy yields important information on the momentum spread of the condensate ground-state wave function and is one of most valuable BEC diagnostics tools [20,21]. The Bragg scattering is also used as a convenient tool for manipulation, e.g. splitting and pushing, condensed atoms. It is instructive to realize that its essence is the same as that of the Raman scattering.

\section{Conclusions}

In this paper we have demonstrated that cold atoms are attractive for nonlinear laser spectroscopy. Main mechanisms involved are Raman resonances between the discrete Zeeman sublevels of trapped atoms and between unbound atomic kinetic states. The latter ones, known as recoil-induced-resonances are shown to be very useful for the trapped-atom diagnostics. We have developed novel spectroscopic method for determination of the momentum distributions, which is non-destructive, highly sensitive and provides multi-dimensional momentum determination in a single measurement. Its potential has been demonstrated by our study of different momentum distributions of atoms in the operating magneto-optical trap. We have also performed pump-probe spectroscopy of ${ }^{85} \mathrm{Rb}$ atoms in an operating MOT equipped with an extra pair of lattice beams. Application of a simple 1D lattice of a standing-wave configuration and non-destructive RIR thermometry allowed studies of the localization efficiency at well-determined atomic temperature, not affected by the lattice. This extension of a standard RIR velocimetry proved very useful for non-destructive diagnostics of 1D-lattice. Raman spectroscopy can also be used for diagnostics of BEC. In such application it is better known as the Bragg spectroscopy and provides important information on the momentum spread of the condensate ground-state wave function. 


\section{References}

[1] See, e.g., R.W. Boyd, Nonlinear Optics, Academic Press, Inc. 1992.

[2] T.M. Brzozowski, M. Brzozowska, J. Zachorowski, M. Zawada, W. Gawlik, Phys. Rev. A 71, 013401 (2005).

[3] J. Guo, P.R. Berman, B. Dubetsky, G. Grynberg, Phys. Rev. A 46, 1426 (1992).

[4] G. Grynberg, J.-Y. Courtois, B. Lounis, P. Verkerk, Phys. Rev. Lett. 72, 3017 (1994).

[5] P. Verkerk, B. Lounis, C. Salomon, C. Cohen-Tannoudji, J. Courtois, Phys. Rev. Lett. 68, 3861 (1992).

[6] M.C. Fischer, A.M. Dudarev, B. Gutiérrez-Medina, M.G. Raizen, J. Opt. B, Quantum Semiclass. Opt. 3, 279 (2001).

[7] P. Verkerk, in: Proc. Int. School of Physics, Varenna, Course CXXXI, Eds. A. Aspect, W. Barletta, R. Bonifacio, IOS Press, Amsterdam 1996, p. 325.

[8] M. Brzozowska, T.M. Brzozowski, J. Zachorowski, W. Gawlik, Phys. Rev. A 72, 061401(R) (2005).

[9] D.R. Meacher, D. Boiron, H. Metcalf, C. Salomon, G. Grynberg, Phys. Rev. A 50, R1992 (1994).

[10] G. Di Domenico, G. Mileti, P. Thomann, Phys. Rev. A 64, 043408-1 (2001).

[11] M. Brzozowska, T.M. Brzozowski, J. Zachorowski, W. Gawlik, Phys. Rev. A 73, 063414 (2006).

[12] M. Gajda, J. Mostowski, Phys. Rev. A 49, 4864 (1994).

[13] M. Gajda, private communication.

[14] P.S. Jessen, C. Gerz, P.D. Lett, W.D. Phillips, S.L. Rolston, R.J.C. Spreeuw, C.I. Westbrook, Phys. Rev. Lett. 69, 49 (1992).

[15] J. Guo, P. Berman, Phys. Rev. A 47, 4128 (1993).

[16] J. Guo, Phys. Rev. A 49, 3934 (1994).

[17] J. Guo, Phys. Rev. A 52, 1458 (1995).

[18] A. Hemmerich, T.W. Hänsch, Phys. Rev. Lett. 70, 410 (1993).

[19] M.R. Andrews, M.-O. Mewes, N.J. van Druten, D.S. Durfee, D.M. Kurn, W. Ketterle, Science 273, 84 (1996).

[20] J. Stenger, S. Inouye, A.P. Chikkatur, D.M. Stamper- Kurn, D.E. Pritchard, W. Ketterle, Phys. Rev. Lett. 82, 4569 (1999).

[21] D.M. Stamper-Kurn, A.P. Chikkatur, A. Gorlitz, S. Inouye, S. Gupta, D.E. Pritchard, W. Ketterle, Phys. Rev. Lett. 83, 2876 (1999). 